\title{
Red supergiants and the past of Cygnus OB2
}

\author{
F. Comerón ${ }^{1, \star}$, A. A. Djupvik ${ }^{2}$, N. Schneider ${ }^{3,4}$, and A. Pasquali ${ }^{5}$ \\ ${ }^{1}$ European Southern Observatory, 3107 Alonso de Córdova, Vitacura, Santiago 19, Chile \\ e-mail: fcomeron@eso.org \\ 2 Nordic Optical Telescope, Apdo 474, 38700 Santa Cruz de La Palma, Spain \\ 3 I. Physik Institut University of Cologne, 50937 Cologne, Germany \\ 4 OASU/LAB-UMR 5804, CNRS, Université Bordeaux 1, 33270 Floirac, France \\ 5 Astronomisches Rechen-Institut, Zentrum für Astronomie der Universität Heidelberg, Mönchhofstr. 12-14, 69120 Heidelberg, \\ Germany
}

Received 6 October 2015 / Accepted 2 December 2015

\section{ABSTRACT}

\begin{abstract}
Context. Red supergiants are the evolved descendants of massive stars with initial masses between 7 and $40 M_{\odot}$. Their brightness makes them easily detectable in the near infrared, making them useful probes of star formation that occurred several tens of Myr ago. Aims. We investigate the past star formation history of Cygnus OB2, the nearest very massive OB association, using red supergiants as a probe. Our aim is to confirm the evidence, found by previous studies, that star formation in the Cygnus OB2 region started long before the latest burst that gave rise to the dense aggregate of early O-type stars that dominate the appearance of the association at present.

Methods. Near-infrared star counts in the Cygnus region reveal moderate evidence for a peak in the areal density of bright, reddened stars approximately coincident with Cygnus OB2. A total of 11 sources are found within a circle of $1^{\circ}$ radius centered on the association, of which 4 are non-supergiants based on existing observations. Near-infrared spectroscopy is presented of the remaining seven candidates, including four that have been already classified as $\mathrm{M}$ supergiants in the literature.

Results. We confirm the presence of seven red supergiants in the region and argue that they are probably physically associated with Cygnus OB2. Their location is roughly coincident with that of the older population identified by previous studies, supporting the scenario in which the main star formation activity in the association has been shifting toward higher Galactic longitudes with time. Their luminosities are compared with the predictions of evolutionary tracks with and without rotation to estimate the mass of their progenitors and ages. In this way, we confirm that massive star formation was already taking place in the area of Cygnus OB2 over 20 Myr ago, and we estimate that the star formation rate in the latest $6 \mathrm{Myr}$ represents a six-fold increase over the massive star formation rate at the time when the progenitors of the current red supergiants were formed.

Conclusions. The Cygnus OB2 association has a history of star formation extending into the past for at least about 20 Myr, probably dovetailing with the general history of star formation in the region that gave rise to other associations like the neighboring Cygnus OB9. The sustained massive star formation history also argues for a long lifetime of the giant molecular complex from which Cygnus OB2 formed, whose remnants constitute the present-day Cygnus X complex.
\end{abstract}

Key words. stars: late-type - open clusters and associations: individual: Cygnus OB2

\section{Introduction}

The Cygnus OB2 association is one of the prime targets for studies of the upper end of the stellar mass function and of the interaction of massive stars with their surrounding molecular environment (e.g. Knödlseder 2003; Schneider et al. 2006; Reipurth $\&$ Schneider 2008). The stellar population of Cygnus OB2 is a showcase of nearly all the varieties of stages that very massive stars undergo in their evolution. Following early studies that hinted at the true richness of this significantly reddened association (Massey \& Thompson 1991; Knödlseder 2000), several efforts have been made to produce an increasingly complete census of the massive population of the association (Comerón et al. 2002; Comerón \& Pasquali 2012; Wright et al. 2015). Parallel to this, studies have investigated aspects such as its spatial extent, star formation history, lower mass content, or possible relationship with neighboring associations (Hanson 2003; Comerón et al. 2008; Drew et al. 2008; Wright et al. 2010; Comerón \& Pasquali 2012; Wright et al. 2014).

\footnotetext{
* Visiting astronomer at the Vatican Observatory.
}

Many of these recent studies (see e.g. Comerón \& Pasquali 2012; Wright et al. 2015, and references therein) show that the star formation history of Cygnus OB2 extends well into the past and that the currently densest and youngest part of the association, where the vast majority of its $\mathrm{O}$ stars are found, is contiguous to, and partly overlapping with, an area where B giants and supergiants abound, indicating that many stars in the association have already evolved away from the main sequence and that the main star-forming sites have been shifting with time. The study of this older component is made difficult by the effects of stellar evolution, and in particular by the fact that its most massive components have already disappeared as supernovae.

A thus far unexplored probe of the massive star formation past of Cygnus OB2 is composed of cool supergiants, the descendants of stars with original masses between $\sim 7$ and $\sim 40 M_{\odot}$ (Hirschi 2010) that reach very low $\left(T_{\text {eff }}<4000 \mathrm{~K}\right)$ photospheric temperatures at some stages of their post-main sequence evolution. If present, red supergiants should be the brightest members of Cygnus OB2 at infrared wavelengths and their spectra should make them easily recognizable; indeed, the high infrared luminosities of these stars make them a useful tool for 
the identification and study of distant, highly reddened clusters (e.g. Messineo et al. 2014; Davies et al. 2007; Clark et al. 2009; Mengel \& Tacconi-Garman 2007). At the same time, their presence and characteristics would set relevant lower limits on the past massive star formation activity of the region.

In this paper we report on seven red supergiants located within or near the boundaries of Cygnus OB2 and consistent with being located at the same distance as the association. Four of them have previously been recognized and classified as $\mathrm{M}$ supergiants, but apparently their possible relationship with Cygnus OB2 had not been taken into consideration. The remaining three are confirmed as red supergiants in this work. By using the most recent synthetic evolutionary tracks we discuss the initial properties and ages of these stars, and are able to extract some conclusions on the distant star formation past of the precursor of the present-day Cygnus OB2 association.

\section{Source selection}

Galactic M supergiants (luminosity classes I-II) cover a range of luminosities spanning nearly two orders of magnitude starting at $\log \left(L / L_{\odot}\right) \simeq 4$ while being confined to a rather narrow range of temperatures between $3500 \mathrm{~K}$ and $4000 \mathrm{~K}$ (Levesque et al. 2005; see Davies et al. 2013, however). Using the synthetic magnitudes derived in the Geneva evolutionary tracks for solar metallicity of Ekström et al. (2012), the faint end in luminosity corresponds to an absolute magnitude $M_{K} \simeq-7.8$, or an unreddened apparent magnitude $K_{0} \simeq 3.0$ at the distance of $1.45 \mathrm{kpc}$ (distance modulus $D M=10.8$; Hanson 2003; Rygl et al. 2012), which we adopt in this study. The bulk of Cygnus OB2 members listed in the recent compilation of Wright et al. (2015) has visual extinctions in the range $4<A_{V}<7$, which using the Cygnus OB2 extinction law derived by Wright et al. (2015) implies $0.35<A_{K}<0.60$. We thus expect $\mathrm{M}$ supergiants in Cygnus OB2 to be very bright at infrared wavelengths, with $K<3.6$.

Several compilations of intrinsic colors of M supergiants can be found in the literature, with published values covering a fairly wide range for a given spectral subtype. Straižys (1987) gave $(J-K)_{0}$ values ranging from 0.91 to 1.20 in the M0I-M4I range, while Ducati et al. (2001) quoted significantly different values, ranging from 0.85 to 0.64 in the same range of spectral types. We regard the values of the compilation of Ducati et al. (2001) in this spectral range with some caution because not only are those values notoriously lower than those of $\mathrm{M}$ spectral types of other luminosity classes, but they also become bluer with increasing spectral subtype and their $(H-K)_{0}$ indices remain negative over the whole $\mathrm{M}$ supergiant spectral range. For the present study we have preferred to use the compilation of Tokunaga (2000), which lists colors ranging from $(J-K)_{0}=0.79$ for a spectral type M0Ia to $(J-K)_{0}=1.07$ for M4Ib.

We have thus searched the 2MASS all-sky point source cata$\log$ to select a sample of candidate $\mathrm{M}$ supergiants in Cygnus OB2 defined by the conditions $K<4.0,(J-K)>1.1$, thus restricting the selection to bright and significantly red, or reddened, stars, and providing a virtually complete sample of $\mathrm{M}$ supergiants at the distance of Cygnus OB2. The brightness limit in $K$ excludes the entire early-type population of Cygnus OB2, whose brightest members are expected to peak at $K_{0}>5$ (Martins \& Plez 2006) except for possible stars with strong near-infrared excess. The search area has been defined as a circle of $1^{\circ}$ radius centered on Galactic coordinates $l=79^{\circ} 8, b=+0^{\circ} 8$ in consistency with the boundaries of Cygnus OB2 proposed in Comerón et al. (2008) and Comerón \& Pasquali (2012), which includes, but is offset from, the current stellar density peak of the association.

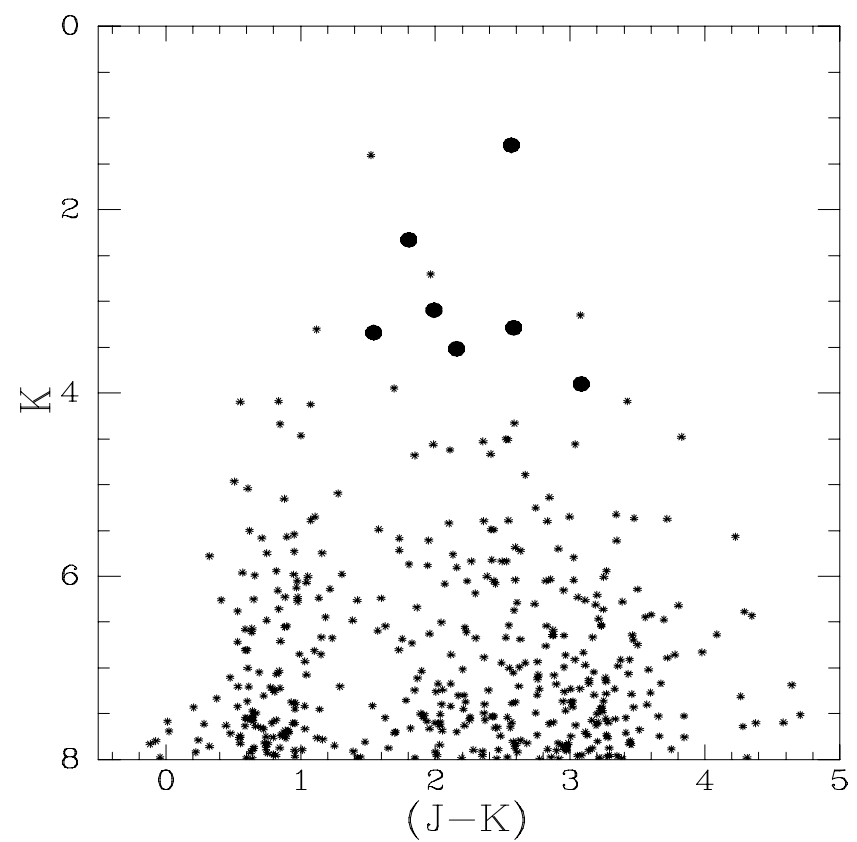

Fig. 1. Color-magnitude diagram of stars with $K<8$ in the field of $1^{\circ}$ radius around $l=79^{\circ} 8, b=+0^{\circ} 8$. The filled circles are the candidate $M$ supergiants selected for spectroscopic observations and, except for the brightest of them, the photometry has been obtained by us as described in Sect. 3.1. Photometry for the remaining stars has been taken from the 2MASS Point Source Catalog.

We note that this definition of the boundaries of Cygnus OB2 differs from that adopted by Wright et al. (2015), which uses a circle of smaller radius centered on the Trapezium-like system VI Cyg 8, very close to the density peak. Our search area thus includes the evolved population, mostly adjacent to the area populated by the newest, early O-type members of the association.

Our selection criteria provided a total of 11 candidates, which are plotted in a near-infrared color-magnitude diagram in Fig. 1. Four of them could be discarded as M supergiant candidates on the basis of previous studies: they are the K5 giant HD 196241 (Haggkvist \& Oja 1970), the M8e Mira variable KZ Cygni (Cameron \& Nassau 1956), the B5Ia+ supergiant VI Cyg 12 (Humphreys 1978) and the Bep star MWC 349 (Brugel \& Wallerstein 1979).

Of the remaining seven candidates, four have previously been confirmed as supergiants through spectroscopy in the visible by other authors: RAFGL 2600 (M1:Iab; Verhoelst et al. 2009), IRAS 20341+4047 (M3I; Imanishi et al. 1996), RAFGL 2605 (M4I:; Grasdalen \& Sneden 1979), and IRAS 20315+4026 (MOI; Imanishi et al. 1996). No classification is available in the literature for the remaining three objects in our selection, which are IRAS 20290+4037, IRAS 20263+4030, and IRAS 20249+4046.

Although individual distance estimates for these stars are not available, the association of red supergiants with Cygnus OB2 is indirectly supported by the fact that the areal density of objects selected by the criteria described above peaks at the position of Cygnus OB2 when a broad range of Galactic longitudes is considered. Applying these selection criteria to areas of $1^{\circ}$ radius that are centered at Galactic longitudes ranging from $l=69^{\circ} 8$ to $l=89^{\circ} 8$, always at a Galactic latitude $b=0^{\circ} 8$, we find an average of 6.6 objects per area, with a standard deviation $\sigma=2.7$. The fact that we find 11 objects within the area of the same radius centered on Cygnus OB2 thus provides some evidence (within 
Table 1. Basic data of the candidate red supergiants.

\begin{tabular}{lcccccll}
\hline \hline Star ID & $\alpha(2000)$ & $\delta(2000)$ & $J$ & $H$ & $K_{S}$ & Sp. type & Name \\
\hline 1 & $20: 33: 23.92$ & $+40: 36: 44.2$ & 6.984 & 4.887 & 3.902 & M0I $^{1}$ & IRAS 20315+4026 \\
2 & $20: 28: 06.19$ & $+40: 40: 06.1$ & 5.675 & 4.420 & 3.518 & - & IRAS 20263+4030 \\
3 & $20: 33: 01.06$ & $+40: 45: 40.4$ & $3.859^{2}$ & $2.026^{2}$ & $1.296^{2}$ & M4I: & RAFGL 2605 \\
4 & $20: 30: 51.53$ & $+40: 48: 08.6$ & 4.884 & 3.814 & 3.342 & - & IRAS 20290+4037 \\
5 & $20: 31: 28.70$ & $+40: 38: 43.3$ & 4.131 & 2.883 & 2.328 & M1:Iab & RAFGL 2600 \\
6 & $20: 26: 43.03$ & $+40: 56: 26.8$ & 5.085 & 3.645 & 3.095 & - & IRAS 20249+4046 \\
7 & $20: 35: 54.73$ & $+40: 57: 40.1$ & 5.869 & 4.198 & 3.288 & M3Iab: & IRAS 20341+4047 \\
\hline
\end{tabular}

Notes. (1) We adopted the classification of Imanishi et al. (1996). Rawlings et al. (2000) suggested that it might be an early star, but this is conclusively ruled out by the $K$-band spectroscopy presented here. ${ }^{(2)}$ Photometry obtained from 2 MASS, uncertain by $\sim 0.3$ mag in each band. The star is too bright for the setup used in our observations, even after strong defocusing. ${ }^{(3)}$ We adopted the classification of Imanishi et al. (1996), which is consistent with the near-infrared spectrum presented here. We note, however, that there are highly discrepant spectral classifications of this object (Rawlings et al. 2000).

the limitations imposed by small-number statistics) of an excess of very bright, red sources coincident with the association. It must be noted that the Galactic longitude range we considered to estimate the density of such sources outside Cygnus OB2 includes other OB associations in Cygnus (Uyaniker et al. 2001) and thus possibly a certain contribution by red supergiants that belong to those associations. On the other hand, the members of our sample of candidate supergiants have infrared colors that imply extinctions in their direction similar to, and in some cases even higher than, those typical of Cygnus OB2 members, which argues against a possible contamination of our sample by foreground late-type red giants. We therefore consider here that that the red supergiants we discussed are all at the same distance as Cygnus OB2.

\section{Observations}

\subsection{Imaging}

The brightness of our targets means that some of them appeared saturated in the 2MASS survey and that their cataloged 2MASS magnitudes were derived from the extrapolation of the unsaturated wings of their point-spread function, whereby the uncertainties in the flux measurements reached $20 \%-30 \%$. We therefore decided to reobserve all the stars in the $J, H$ and $K_{\mathrm{S}}$-bands using the CAIN-3 near-infrared camera at the $1.5 \mathrm{~m}$ Carlos Sánchez telescope at the Observatorio del Teide (Tenerife, Canary Islands, Spain). The observations were carried out in service mode on the nights of 19 May and 2 June 2015. To avoid saturation the telescope was defocused before each observation and counts over the resulting stellar image were checked to fall within the linear regime of the detector. Ten stacks of images were obtained for each star, each consisting of eight individual exposures of $0.25 \mathrm{~s}$ integration time, with small $\left(15^{\prime \prime}\right)$ telescope offsets in between. The images were flat-fielded and sky-subtracted using as background sky the image obtained by combining the ten stacks uncorrected for the telescope dithers, using sigma clipping to remove the stellar images from the individual frames. Four bright stars with well-determined magnitudes in 2MASS near our targets were observed just before and after to provide accurate flux calibration. Aperture photometry was then performed on the reduced images of all the sources using an oversized aperture to include the entire flux from the defocused images. Contamination in such large apertures proved to be negligible, as both our targets and the stars selected for flux calibration are by far the brightest objects in their fields of view at near-infrared wavelengths. The photometry of our targets is presented in Table 1.

\subsection{Spectroscopy}

All seven stars in our sample, including those for which spectral classifications as supergiants were already available in the literature, were observed in the near-infrared $K$-band using the NOTCam array camera and spectrograph at the Nordic Optical Telescope (NOT) at the Observatory of Roque de los Muchachos (La Palma, Canary Islands, Spain). The spectra were obtained on the nights of 1 June, 2 August, and 2 September 2015. The A0V star HIP 99719, observed with the same setup as our targets just before and after them, was used as a calibrator to remove telluric features.

The NOTCam spectrograph with its Hawaii-1 HgCdTe array was used with the wide-field camera $\left(0.234^{\prime \prime} /\right.$ pix $)$, Echelle grism \#1, a $0.6^{\prime \prime}$ wide slit, and the MKO $K$-band filter used as an order sorter. With this setup the dispersion is $4.1 \AA /$ pix, giving a spectral resolution $\lambda / \Delta \lambda \sim 2100$ at $2.2 \mu \mathrm{m}$.

The data were obtained in an A-B-B-A dithering pattern along the slit. The very bright targets needed exposure times in the range 3.6 to $12 \mathrm{~s}$ per dither position, while for the fainter telluric standard calibrator we used $60 \mathrm{~s}$, all obtained with the sample-up-the-ramp readout mode. When acquiring the stars on the slit, we added a narrow-band filter in the beam to avoid heavy saturation of the detector, which otherwise could produce persistency effects in the spectra. Halogen lamp flats were obtained in situ to better correct for fringing.

One-dimensional spectra of our targets and telluric calibration star were extracted using dedicated scripts based on IRAF tasks and wavelength calibrated using the sky emission lines as a built-in calibration source (Oliva \& Origlia 1992). The target spectra were ratioed by that of the telluric calibration star and multiplied by an artificially generated blackbody spectrum at $T=10000 \mathrm{~K}$, approximately representing the spectrum of the A0V telluric calibrator, to obtain a relative flux calibration.

\section{Results}

\subsection{Spectra classification}

Figure 2 displays the spectra of all our M supergiant candidates, including those for which a classification is already available in the literature. 


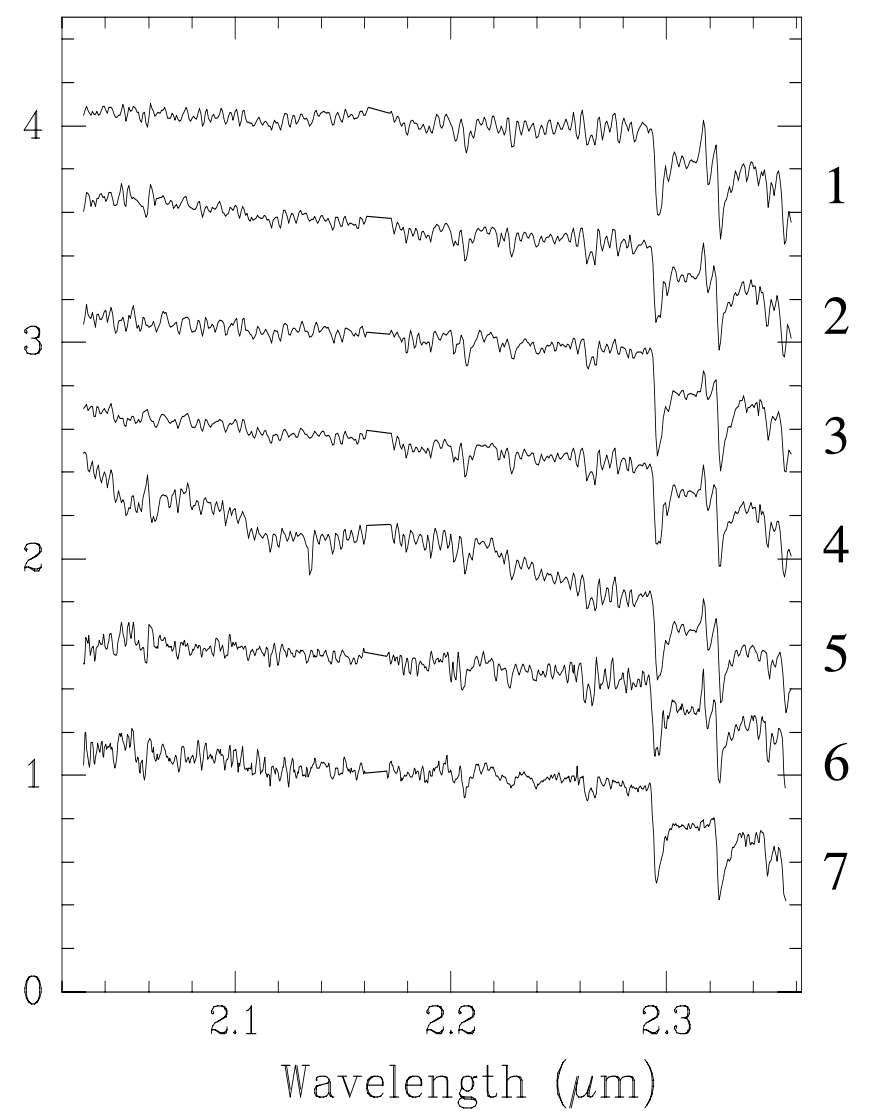

Fig. 2. $K$-band spectra of the candidate Cygnus OB2 M supergiants obtained with NOTCam at the Nordic Optical Telescope. The appearance of the spectra is dominated by the CO bands longward from $2.293 \mu \mathrm{m}$, which reach their maximum strength in red supergiants. The features of $\mathrm{NaI}(2.207 \mu \mathrm{m})$ and $\mathrm{CaI}(2.263 \mu \mathrm{m})$, also prominent in red supergiants, are clearly visible in all the spectra. A technical problem affected the response of the detector during the observations of star 5, therefore the shape of the continuum of this star is not reliably represented in the spectrum.

Table 2. Equivalent widths of spectral features.

\begin{tabular}{lcccc}
\hline \hline Star ID & $\begin{array}{c}E W(\mathrm{NaI}) \\
\AA\end{array}$ & $\begin{array}{c}E W(\mathrm{CaI}) \\
\AA\end{array}$ & $\begin{array}{c}E W(\mathrm{CO}) \\
\AA\end{array}$ & Sp. type (IR) ${ }^{1}$ \\
\hline 1 & 4.3 & 2.9 & 25.2 & \\
2 & 4.8 & 3.5 & 23.7 & M1I \\
3 & 4.5 & 3.5 & 31.1 & M0I \\
4 & 4.8 & 4.1 & 23.9 & M0I \\
5 & 4.3 & 4.7 & 28.1 & M3I \\
6 & 4.7 & 4.6 & 22.6 & K7I \\
7 & 4.1 & 2.5 & 29.8 & M4I \\
\hline
\end{tabular}

Notes. ${ }^{(1)}$ Spectral type estimated from the equivalent width of the first $\mathrm{CO}(2-0)$ band.

M supergiants can be distinguished from other cool luminous stars through several spectroscopic features in the $K$-band (Kleinmann \& Hall 1986; Wallace \& Hinkle 1996, 1997; Lançon \& Wood 2000). The most outstanding one is the series of CO bandheads starting at $\lambda=2.293 \mu \mathrm{m}$. This is characteristic of cool photospheres, which reach their maximum depth in M supergiants. The features produced by some neutral species are also prominent, most notably $\mathrm{NaI}$ and $\mathrm{CaI}$ at $2.207 \mu \mathrm{m}$ and $2.263 \mu \mathrm{m}$ respectively. Table 2 lists the equivalent widths
$(E W)$ of those features that were obtained using passbands centered on them. The reference continuum was taken on both sides of the feature and interpolated, with the exception of the $\mathrm{CO}(\lambda=2.293 \mu \mathrm{m})$ molecular band for which two reference continuum intervals on the shorter wavelength side were used for extrapolation, because there is no suitable continuum near the red edge of the feature. The positions and widths of the passbands used to measure the equivalent widths of the features and their chosen continuum reference points are the same as described in Comerón et al. (2004). Uncertainties in the equivalent width measured in this way were estimated by repeating the measurements with different choices of the continuum level. We thus estimate errors $\Delta E W(\mathrm{CO}) \simeq 1.1 \AA$, and $\Delta E W(\mathrm{NaI}) \simeq$ $\Delta E W(\mathrm{CaI}) \simeq 0.8 \AA$.

Equivalent widths of the $\mathrm{CO}$ features of our candidates are consistently in the range of the galactic supergiants presented by Lançon \& Wood (2000). This same range is also occupied by oxygen-rich long-period variables, but the latter usually display deep and very broad water vapor absorption bands whose wings dominate the appearance of the $K$-band spectrum at wavelengths shorter than $\sim 2.1 \mu \mathrm{m}$. These are clearly absent from our spectra. On the other hand, all the measurements of $E W(\mathrm{CO})$ of red giants in the sample considered by Davies et al. (2007) fall below those that we obtain in our sample, even including red giants of types M7 or later, thus giving us further confidence that all the stars in our sample are true supergiants.

We used our spectra to estimate spectral types according to their correlation with $E W(\mathrm{CO})$ for supergiants presented by Davies et al. (2007). The blue edge of the passband we used to define $E W(\mathrm{CO})$ is at a slightly shorter wavelength $(2.289 \mu \mathrm{m})$ than the band used by these authors. This is nevertheless necessary to account for the effect of our lower spectral resolution, which degrades the sharp drop in flux at the $\mathrm{CO}$ bandhead and spreads it toward shorter wavelengths in our spectra. The bandpass we adopted thus ensures that the first CO band is fully enclosed in it.

The spectral types we estimate from $E W(\mathrm{CO})$ are listed in Table 2. As shown in Fig. 2 of Davies et al. (2007) there is considerable spread in the $E W(\mathrm{CO})$ vs. spectral type relation, especially among the latest-type supergiants. Spectral types estimated in this way can differ from the spectral classification in the visible by up to four or five subclasses. However, a comparison between the spectral types estimated from $E W(\mathrm{CO})$ and those listed in Table 1 for the four stars for which classifications based on the visible spectrum exist in the literature indicate a fairly close match, with the largest discrepancy, in the case of Star 5, amounting to only two subclasses.

\subsection{Space distribution}

Figure 3 shows the location of the M supergiants projected on the area of Cygnus OB2, and different classes of previously known early-type stars indicated. The latter come from the survey of Comerón \& Pasquali (2012), which is known to represent an incomplete census of early-type objects in the region. However, the uniform photometric selection criterion used in that work leads to an approximately unbiased sample that is useful in the present case for a comparison of the spatial distribution of cool supergiants with those of other objects, particularly giant and supergiant B-type stars. Like other representatives of the evolved population of Cygnus OB2, we find all our cool supergiants to lie adjacent to the density peak dominated by $\mathrm{O}$ stars, in the region where evolved B giants and A stars (Drew et al. 2008) tend to be located, thus confirming that star formation has been progressing 


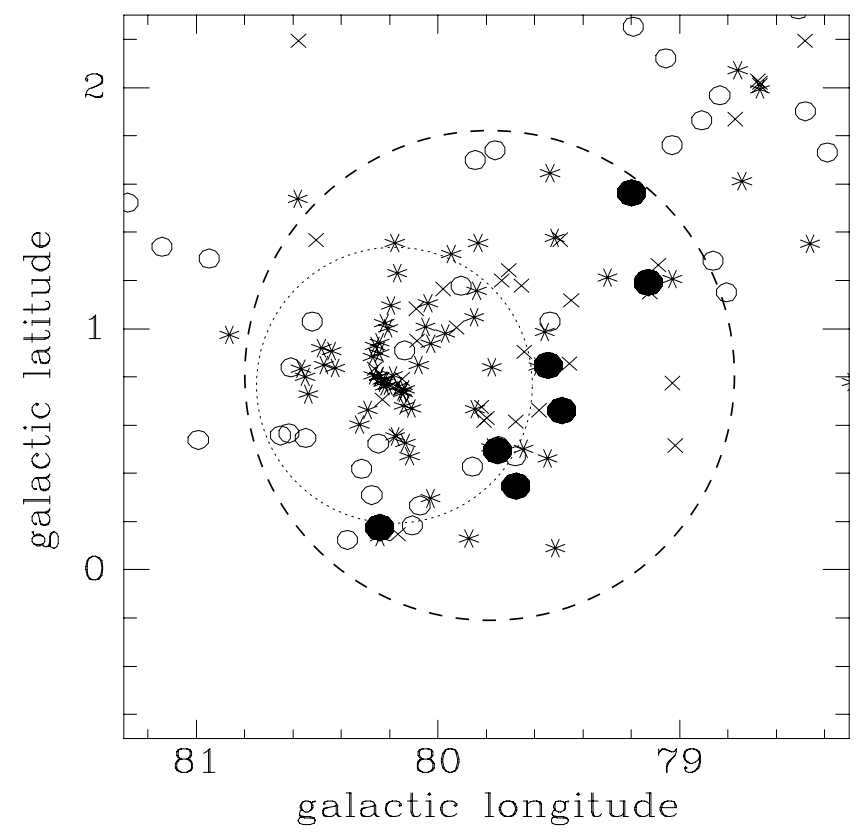

Fig. 3. Spatial distribution of the $M$ supergiants discussed in this study (filled circles). Other symbols represent stars from the survey of Comerón \& Pasquali (2012), which correspond to O-type stars (asterisks), main sequence B stars (crosses) and B-type giants and supergiants (open circles). The dashed line delimits the boundary of Cygnus OB2 as adopted by Comerón \& Pasquali (2012) and is the area where we have searched for $\mathrm{M}$ supergiant candidates. For reference, the area studied in detail by Wright et al. (2015) is indicated by the smaller, dotted circle.

toward higher Galactic longitudes in the time elapsed between the birth of the progenitor population of the currently observed cool supergiants and the most recently formed early-type O stars. For reference, the area covered by the compilation of Wright et al. (2015) is also depicted in Fig. 3. The latter includes a smaller area where Wright et al. (2014) have studied the properties of the lower-mass, X-ray emitting population, which is also dominated by a very young (3-5 Myr) component.

\subsection{Evolutionary stage}

Both the age at which a star reaches the red supergiant phase, and the luminosity during that phase, are related to the initial mass of its progenitor. Rotation and mixing in the interior of the star also have great importance in determining the post-main sequence evolution and the path followed by the star in the temperatureluminosity diagram (e.g. Ekström et al. 2012).

We adopted the average $(J-K)_{0}$ color of M supergiants in the compilation of Tokunaga (2000) to estimate the extinction toward all our targets. Given the uncertainties in the classification of even previously known and spectroscopically classified objects, together with the differences among different derivations of the average infrared colors for a given spectral type as discussed above, the resulting adopted value is $(J-K)_{0}=$ $0.93 \pm 0.15$, which translates into an uncertainty of 0.27 mag in the $K$-band extinction $A_{K}$ using the Cygnus OB2 extinction law from Wright et al. (2015).

We derived $K$-band bolometric corrections $B C_{K}$ as a function of the effective temperature $T_{\text {eff }}$ by using the values of the absolute bolometric magnitude $M_{\text {bol }}, K$-band absolute magnitude $M_{K}$, and effective temperature $T_{\text {eff }}$ from the solarmetallicity evolutionary tracks of red supergiants with $10 M_{\odot}$
Table 3. Derived extinction, temperature and luminosity.

\begin{tabular}{lccc}
\hline \hline Star ID & $A_{V}$ & $T_{\text {eff }}(\mathrm{K})$ & $\log L\left(L_{\odot}\right)$ \\
\hline 1 & 14.4 & 3745 & 4.06 \\
2 & 8.2 & 3790 & 4.02 \\
3 & 10.9 & 3450 & 4.89 \\
4 & 4.1 & 3790 & 3.95 \\
5 & 5.8 & 3605 & 4.36 \\
6 & 7.1 & 3830 & 4.17 \\
7 & 11.0 & 3535 & 4.15 \\
\hline
\end{tabular}

initial mass from Ekström et al.( 2012). For the effective temperatures we used the same calibration as in Davies et al. (2007), which is based on the spectral type vs. temperature calibration of Levesque et al. (2005) for Milky Way supergiants. The validity of this calibration has recently been challenged by Davies et al. (2013), who have estimated effective temperatures of cool supergiants in the Large and Small Magellanic Clouds based on fits to MARCS model atmospheres (Gustafsson et al. 2008). Davies et al. (2013) find discrepant results when the temperature is derived based on either the fit to the $\mathrm{TiO}$ bands that dominate the spectroscopic classification in the visible, or to the overall continuum. The authors also discuss shortcomings in the modeling of the $\mathrm{TiO}$ absorption that are likely to underestimate $T_{\text {eff }}$ when the latter are used for the fit. The problem is also indicated by Levesque et al. (2007), who found that the temperatures of Magellanic Cloud supergiants are significantly below evolutionary model predictions at the appropriate metallicity. The comparison between Small and Large Magellanic Clouds presented by Davies et al. (2013) even suggests that the discrepancies increase with increasing metallicity, hinting at underestimates of $T_{\text {eff }}$ that might reach over $600 \mathrm{~K}$ for early M-type supergiants in the Milky Way when temperatures are based on spectroscopic classifications in the visible. Since our spectral type estimates are based on a calibration of the CO band depth with respect to spectroscopic classifications in the visible as discussed in Sect. 4.1, such caveats are equally applicable to the $T_{\text {eff }}$ vs. spectral type relation adopted for the spectral types listed in Table 2. On the other hand, recent results on the determination of fundamental parameters of cool supergiants based on spatially resolved interferometric observations tend to support the $T_{\text {eff }}$ vs. spectral type relation of Levesque et al. (2005) for nearby Galactic red supergiants (e.g. Ohnaka et al. 2013; Arroyo-Torres et al. 2013, 2015). By deriving $T_{\text {eff from the measured diameter }}$ at a variety of wavelengths, the dependency on the adopted atmosphere model is greatly reduced with respect to the synthetic atmosphere fits on which the method of Levesque et al. (2005) is based.

Table 3 lists the extinctions, adopted temperatures, and luminosities thus derived for our sample. We used $A_{K}=0.0845 A_{V}$ in agreement with Wright et al. (2015). We assumed an uncertainty temperature $\Delta T_{\mathrm{eff}} \sim 200 \mathrm{~K}$, which brackets the uncertainties in the Levesque et al. (2005) $T_{\text {eff }}$ vs. spectral type relation, as well as an assumed uncertainty of two subclasses in the $E W(\mathrm{CO})$ vs. spectral type relation discussed in Sect. 4.1. The temperature uncertainty results in an uncertainty of $\simeq 0.15 \mathrm{mag}$ in $B C_{K}$. However, given the importance of the latter quantity in deriving the initial mass of the red supergiant precursors, we adopted $\Delta B C_{K}=0.30$ to account for systematic effects derived from a possible erroneous choice of the temperature scale. The adopted uncertainty in the luminosity is then computed as the quadratic sum of the uncertainties in the photometry, the extinction, and the bolometric correction. 


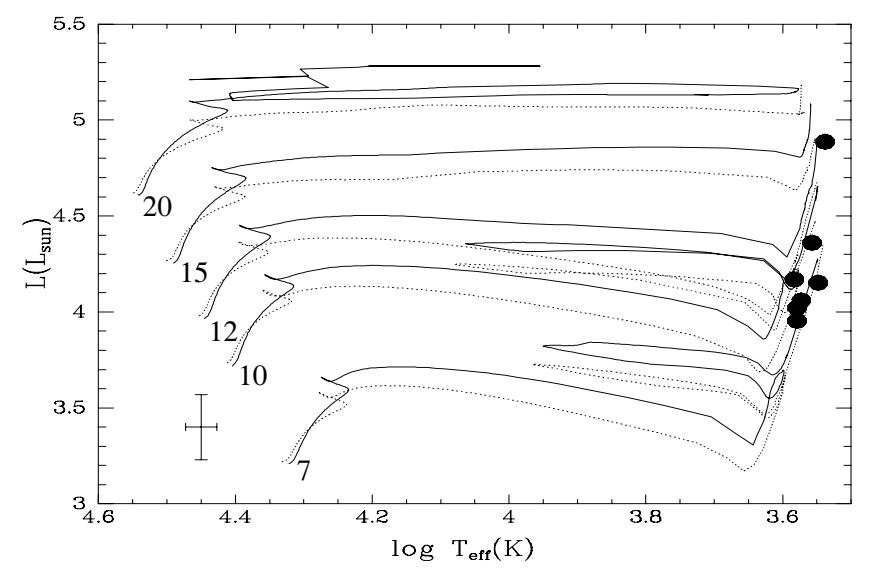

Fig. 4. Location of our Cygnus OB2 M supergiant candidates with respect to the Geneva evolutionary tracks, produced using the SYCLIST application (Georgy et al. 2014) and corresponding to an initial rotational velocity $v=0.4 v_{\text {crit }}$ (solid lines) and to non-rotating models (dotted lines). See Fig. 5 for the identification of the individual stars.

Figure 4 shows the location of our seven stars in the temperature-luminosity diagram, where Geneva evolutionary tracks for models of different masses with and without rotation (Ekström et al. 2012) are also plotted. For masses below $\sim 12 M_{\odot}$ (the precise limit depends on the initial rotation rate), moderately rotating or non-rotating stars evolve to cool temperatures and first become red supergiants at the beginning of the He burning phase, after which their temperatures temporarily increase, performing a blue loop in the temperature-luminosity diagram before returning to low surface temperatures and ending their lives as red supergiants again before they explode as type II supernovae. For a $10 M_{\odot}$ star, the duration of the red supergiant phase previous to the blue loop, which we define as the time spent with a photospheric temperature $T_{\text {eff }}<4000 \mathrm{~K}$, lasts nearly 2 Myr (again depending on the initial rotation rate), which is much longer than the time spent in the final, post-blue loop red supergiant stage. The evolution becomes simpler to describe for stars between $\sim 12 M_{\odot}$ and $\sim 20 M_{\odot}$, which do not undergo the blue loop excursion. After reaching the red supergiant stage, these stars remain there until the end of their lives, for a time span ranging from somewhat less than 1 Myr to somewhat more than 2 Myr, depending on the initial mass and rotation velocity.

An examination of the locations of our sample in the temperature-luminosity diagram suggests that the five least luminous stars in our sample (numbers $1,2,4,6$, and 7) are located near the path followed by pre-blue loop cool supergiants with initial masses around $\sim 10 M_{\odot}$, whereas star 5 probably has a more massive progenitor of $\sim 12 M_{\odot}$ and star 3 is the most massive, with an initial mass of $\sim 15 M_{\odot}$. The effects of different initial rotational velocities for initial masses of $10 M_{\odot}$ is illustrated in Fig. 5, where three tracks that correspond to no rotation, intermediate rotation, and rotation at $90 \%$ of the critical velocity $v_{\text {crit }}$ are illustrated. This shows that very fast rotation can suppress the blue loop as seen in Fig. 5, but the duration of the cool supergiant phase is still comparable to the pre-blue loop stage of slower rotating stars of the same initial mass.

The presence of five relatively low-mass red supergiants has important consequences for the star formation history in or near Cygnus OB2, given the relatively long duration of their evolution before they first enter the red supergiant domain. The Geneva evolutionary tracks indicate that a non-rotating star with initial mass of $10 M_{\odot}$ enters the red supergiant phase at an age

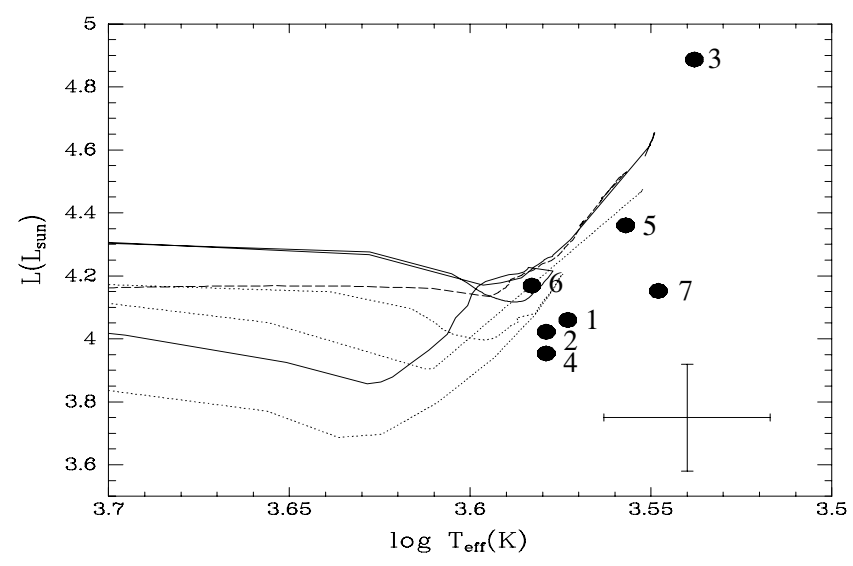

Fig. 5. Same as Fig. 4, showing the effects of rotation for a $M=10 M_{\odot}$ star in the proximities of the red supergiant phase. The curves correspond to an initial rotational velocity $v=0.4 v_{\text {crit }}$ (solid line), $v=0.9 v_{\text {crit }}$ (dashed line), and a non-rotating model (dotted line).

of $20.9 \mathrm{Myr}$, and that rotation delays this phase until the star becomes 25.2 Myr old if the star is rotating at $90 \%$ of $v_{\text {crit }}$. The existence of such relatively low-luminosity red supergiants with initial masses of $\sim 10 M_{\odot}$ thus indicates that massive star formation was already proceeding in the region over $20 \mathrm{Myr}$ ago. At higher masses, star 5 probably comes from a progenitor formed between 15.4 Myr and 21.6 Myr ago, while that of star 3 formed between 11.5 an 15.6 Myr ago.

Our selection criteria should in principle be sensitive to red supergiants with $M_{\text {bol }}>-5.15$ and thus to masses below $10 M_{\odot}$, but we did not find any candidates with these characteristics. This might be an indication that the onset of star formation in the region took place less than $\sim 25 \mathrm{Myr}$ ago, thus implying that stars with masses below $10 M_{\odot}$, despite being more abundant by virtue of the shape of the mass function, have not had time to reach the red supergiant phase. Given the small numbers involved, the uncertainties in the derivation of the intrinsic properties, and the uncertainties in the model evolutionary tracks this conclusion cannot be firmly established, however, and needs to be confirmed by future work.

\subsection{Star formation rates present and past}

A comparison between the catalog of early-type stars in the presently densest part of Cygnus OB2 produced by Wright et al. (2015) and the number of cool supergiants that we find allows us to obtain a rough estimate of the evolution of the star formation rate in the region. Wright et al. (2015) estimated their catalog to be complete for stars with masses between $20 M_{\odot}$ and $40 M_{\odot}$ and younger than $6 \mathrm{Myr}$. The individual masses and ages listed by these authors show 31 stars with these characteristics in an area of one square degree considered in their study. Using the initial mass function that they derive, $\zeta(M) M \propto M^{-2.3} \mathrm{~d} M$, this implies the existence of $\sim 53$ stars formed during the same interval in the mass range $10 M_{\odot}<M<15 M_{\odot}$, where we find the progenitor masses of the cool supergiants identified by us. This can be written as

$$
\begin{aligned}
N\left(10 M_{\odot}<M<\right. & \left.15 M_{\odot}, t<6 \mathrm{Myr}\right)= \\
& A_{1} \int_{M=10 M_{\odot}}^{15 M_{\odot}} \zeta(M) \mathrm{d} M \int_{t=-6 \mathrm{Myr}}^{0} \Psi(t) \mathrm{d} t
\end{aligned}
$$


where $A_{1}$ is the area of 1 square degree considered by Wright et al. (2015), $\Psi(t)$ is the average star formation rate per unit area, and we define $t=0$ at present.

The number of stars with masses between $10 M_{\odot}$ and $15 M_{\odot}$ undergoing the red supergiant stage at present can be described through a similar expression:

$$
\begin{aligned}
N_{\mathrm{SG}}\left(10 M_{\odot}<M<15 M_{\odot}\right)= & \\
& A_{2} \int_{v=0}^{v_{\text {crit }}} \phi(v) \mathrm{d} v \int_{M=10 M_{\odot}}^{15 M_{\odot}} \zeta(M) \mathrm{d} M \int_{t=-t_{2}(M, v)}^{-t_{1}(M, v)} \Psi(t) \mathrm{d} t
\end{aligned}
$$

where $A_{2}$ is the area of our search ( $\pi$ square degrees); $\phi(v)$ is the distribution function of initial rotation velocities, which we assume to be uniform; and $t_{1}(M, v), t_{2}(M, v)$ are the ages of a star of mass $M$ and initial rotational velocity $v$ at the time of entering and ending the red supergiant phase, respectively. The evolutionary tracks at solar metallicity of Ekström et al. (2012) were used to obtain $t_{1}(M, v)$ and $t_{2}(M, v)$.

If we assume for simplicity that $\Psi(t)$ has stayed constant at $\Phi(t)=\Phi_{1}$ over the past $6 \mathrm{Myr}$ in which the young population characterized by Wright et al. (2015) formed, and that it had a different value $\Phi(t)=\Phi_{2}$ in the past when the progenitor populations of the present red supergiants formed, the ratio of Eqs. (1) and (2) yields

$$
\frac{N\left(10 M_{\odot}<M<15 M_{\odot}, t<6 \mathrm{Myr}\right)}{N_{\mathrm{SG}}\left(10 M_{\odot}<M<15 M_{\odot}\right)}=\frac{53}{7}=3.94 \frac{\Psi_{1} A_{1}}{\Psi_{2} A_{2}}
$$

thus leading to $\Psi_{1} \sim 6 \Psi_{2}$, this is, the star formation rate in the region has increased by a factor $\sim 6$ since the progenitors of the currently observed red supergiants were born. Furthermore, as noted in Sect. 4.2 the increasing star formation rate has been accompanied by a progression of the main star-forming locations toward higher Galactic longitudes. The study of the spatial distribution and clustering properties of the youngest dynamically unevolved parts of the association carried out by Wright et al. (2014) shows that recent star formation has resulted in relatively low-density aggregates, as opposed to clusters, with no indication of mass segregation. As noted by Wright et al. (2014), this picture in which recent star formation has taken place at separate locations rather than as a single spatially confined burst suggests that the star formation history in the Cygnus OB2 region has been a succession of localized episodes extended over space, and probably also in time although the study of Wright et al. (2014) is restricted by design to the youngest population. This picture is consistent with the results we obtain when considering a much larger area, where the evidence for star formation extended in time becomes clear.

It may be noted that the duration of the star formation history in the Cygnus OB2 region that we infer is on the same order as the typical lifetime of giant molecular clouds in our Galaxy and others, which has been estimated to be around 20-30 Myr from numerical simulations and from direct observations (e.g. Blitz et al. 2007; Murray 2011). On the other hand, the abundant molecular gas reservoir still existing in the Cygnus X complex $\left(\sim 10^{6} M_{\odot}\right.$; Schneider et al. 2006) that surrounds the Cygnus OB2 association leads to the expectation that star-forming activity in it will still extend some Myr into the future. Taking this together with the fact that massive star formation and its associated disruptive feedback were present already over $20 \mathrm{Myr}$ ago, we suggest that the parental giant molecular cloud whose remnants compose Cygnus $\mathrm{X}$ today may have had properties that led to a lifetime somewhat longer than typical.

\section{Conclusions}

The work presented here shows indications of an excess of very bright, red sources in the general direction of Cygnus OB2 that we ascribe to the presence of red supergiants at the distance of the association. Some of these sources have been found to be other types of objects by previous studies, but spectral classifications in the literature complemented with new observations presented here show that seven such stars are indeed late-type supergiants. The study of this sample leads us to the following conclusions:

- The red supergiants lie in the region immediately adjacent to Cygnus OB2 and partly overlap it. In this region, where previous studies have revealed the presence of early-B stars that left the main sequence, as well as other types of stars that indicate an average age older than that of the current main concentration of $\mathrm{O}$ stars that form the youngest part of the association. The location of the reported population of red supergiants is thus consistent with previous findings that have suggested that star formation has proceeded from lower Galactic longitudes toward the direction where the densest and youngest part of Cygnus OB2 is found at present.

- The presence of red supergiants implies that star formation in the Cygnus OB2 region started at least 20 Myr ago. Indications of a low-luminosity cutoff in this population of red supergiants suggest, however, that star formation started not much earlier than this estimate.

- A comparison to the census of the younger ( $<6 \mathrm{Myr}$ ) population of Cygnus OB2 allowed us to carry out a crude estimate of the increase in the star formation rate in the region with time. We find that the massive star formation rate has increased by a factor of $\sim 6$ between the present time (represented by stars younger than $6 \mathrm{Myr}$ ) and the time when the progenitors of the current population of red supergiants formed.

Our results provide new support of the view that has emerged from studies carried out over the past decade. This view holds that the star formation history in the Cygnus OB2/Cygnus X region started well before the last burst that gave rise about $3 \mathrm{Myr}$ ago to the compact aggregate of early O-type stars that currently dominate the $\mathrm{OB}$ association. Our results extends this history even further into the past. On the other hand, it must be kept in mind that the existence of neighboring $\mathrm{OB}$ associations such as Cygnus OB1 and OB9, which lie approximately at the same distance, have certainly also played a role in the overall evolution of the Cygnus region, thus highlighting the complexities of a star formation history that may be revealed by future studies of tracers of its older stellar population.

Acknowledgements. This paper is based on observations collected at the Observatorio del Teide, operated by the Instituto de Astrofísica de Canarias (IAC), in the island of Tenerife (Canary Islands, Spain); and at the Nordic Optical Telescope at the Observatory of Roque de Los Muchachos, in the Island of La Palma (Canary Islands, Spain). We are thankful to the astronomers in charge of service mode observing at the Observatorio del Teide for the careful execution of our program. F.C. warmly thanks the Specola Vaticana for their hospitality during the time when much of this work was done. F.C. and A.P. acknowledge support from the DFG Research Centre SFB 881 "The Milky Way System" through project B5. The authors are grateful to the referee, Ben Davies, for very useful comments that helped improve the quality of this paper. This research has made use of the SIMBAD database operated at CDS, Strasbourg, France. It also makes use of data products from the Two Micron All Sky Survey, which is a joint project of the University of Massachusetts and the Infrared Processing and Analysis Center/California Institute of Technology, funded by the National Aeronautics and Space Administration and the National Science Foundation. 


\section{References}

Arroyo-Torres, B., Wittkowski, M., Marcaide, J. M., \& Hauschildt, P. H. 2013, A\&A, 554, A76

Arroyo-Torres, B., Wittkowski, M., Chaivassa, A., et al. 2015, A\&A, 575, A50

Blitz, L., Fukui, Y., Kawamura, A., et al. 2007, in Protostars and Planets V., eds.

B. Reipurth, D. Jewitt, \& K. Keil, Univ. of Arizona Press

Brugel, E. W., \& Wallerstein, G. 1979, ApJ, 229, L23

Cameron, D., \& Nassau, J. J. 1956, ApJ, 124, 346

Clark, J. S., Negueruela, I., Davies, B., et al. 2009, A\&A, 498, 109

Comerón, F., \& Pasquali, A. 2012, A\&A, 543, A101

Comerón, F., Pasquali, A., Rodighiero, G., et al. 2002, A\&A, 389, 874

Comerón, F., Torra, J., Chiappini, C., et al. 2004, A\&A, 425, 489

Comerón, F., \& Pasquali, A., Figueras, F., \& Torra, J. 2008, A\&A, 486, 453

Davies, B., Figer, D. F., Kudritzki, R.-P., et al. 2007, ApJ, 671, 781

Davies, B., Kudritzki, R.-P., Plez, B., et al. 2013, ApJ, 767, 3

Drew, J. E., Greimel, R., Irwin, M. J., \& Sale, S. E. 2008, MNRAS, 387, 08

Ducati, J. R., Bevilacqua, C. M., Rembold, S. B., \& Ribeiro, D. 2001, AJ, 558, 309

Ekström, S., Georgy, C., Eggenberger, P., et al. 2012, A\&A, 537, A146

Georgy, C., Granada, A., Ekström, S., et al. 2014, A\&A, 566, A21

Grasdalen, G. L., \& Sneden, C. 1979, PASP, 91, 337

Gustafsson, B., Edvardsson, B., Eriksson, K., et al. 2008, A\&A, 486, 951

Haggqvist, L., \& Oja, T. 1970, A\&AS, 1, 199

Hanson, M. M. 2003, ApJ, 597, 957

Hirschi, R. 2010, ASP Conf. Ser., 425, 13

Humphreys, R. M. 1978, ApJS, 38, 309

Imanishi, M., Sasaki, Y., \& Goto, M. 1996, AJ, 112, 235

Kleinmann, S. G., \& Hall, D. N. B. 1986, ApJS, 62, 501

Knödlseder, J. 2000, A\&A, 360, 539
Knödlseder, J. 2003, in A Massive Star Odyssey: From Main Sequence to Supernova, eds. K. van der Hucht, A. Herrero, \& C. Esteban (San Francisco: ASP), IAU Symp., 212

Lançon, A., \& Wood, P. R. 2000, A\&AS, 146, 217

Levesque, E. M., Massey, P., Olsen, K. A. G., et al. 2005, ApJ, 628, 973

Levesque, E. M., Massey, P., Olsen, K. A. G., et al. 2007, ApJ, 667, 202

Martins, F., \& Plez, B. 2006, A\&A, 457, 637

Massey, P., \& Thompson, A. B. 1991, AJ, 101, 1408

Mengel S., \& Tacconi-Garman, L. E. 2007, A\&A, 466, 151

Messineo, M., Qingfeng, Z., \& Ivanov, V. D. 2014, A\&A, 571, A43

Murray, N. 2011, ApJ, 729, 133

Ohnaka, K., Hofmann, K.-H., \& Schertl, D. 2013, A\&A, 555, A24

Oliva, E., \& Origlia, L. 1992, A\&A, 254, 466

Rawlings, M. G., Adamson, A. J., \& Whittet, D. C. B. 2000, ApJS, 131, 531

Reipurth, B., \& Schneider, N. 2008, in Handbook of Star Formation Regions, ed. B. Reipurth, ASP Monograph (San Francisco: ASP)

Rygl, K. L. J., Brunthaler, A., Sanna, A., et al. 2012, A\&A, 539, A79

Schneider, N., Bontemps, S., Simon, R., et al. 2006, A\&A, 458, 855

Straižys, V. 1987, Bull. Obs. Vilnius, 78, 43

Tokunaga, A. T. 2000, in Allen's Astrophysical Quantities, ed A. N. Cox (New York: AIP Press)

Uyaniker, B., Fürst, E., Reich, W., Aschenbach, B., \& Wielebinski, R. 2001, A\&A, 371, 675

Verhoelst, T., van der Zypen, N., Hony, S., et al. 2009, A\&A, 498, 127

Wallace, L., \& Hinkle, K. 1996, ApJS, 107, 312

Wallace, L., \& Hinkle, K. 1997, ApJS, 111, 445

Wright, N. J., Drake, J. J., Drew, J. E., \& Vink, J. S. 2010, ApJ, 713, 871

Wright, N. J., Parker, R. J., Goodwin, S. P., \& Drake, J. J. 2014, MNRAS, 438, 639

Wright, N. J., Drew, J. E., \& Mohr-Smith, M. 2015, MNRAS, 449, 741 